www.nature.com/clinicalpractice/onc

\title{
Lessons learned from lung cancer chemoprevention trials
}

\author{
Edward S Kim
}

Almost a century ago, fewer than several hundred cases of lung cancer were reported in the literature. Now, lung cancer is a worldwide epidemic, with an estimated annual incidence of over 1.2 million cases and over 1.1 million deaths. ${ }^{1}$ Treatment for advanced lung cancer has improved only modestly, and screening high-risk individuals has failed to produce meaningful improvements in survival. Smoking is the major cause of, and risk factor for, lung cancer; however, smoking cessation programs aimed at reducing lung cancer risk have had limited success. For the field of chemoprevention to move forward, we must learn from our clinical experiences.

Large-scale chemoprevention trials have provided exciting evidence of significant risk reductions for colorectal, breast and prostate cancers. ${ }^{2-4}$ However, large-scale lung cancer chemoprevention trials have yet to show any reduction in lung-cancer risk. ${ }^{5-7}$ The complexity of lung-cancer biology, and the array of multiple defects and epithelial changes that result from carcinogen exposure, are difficult challenges for any approach that aims to reduce the burden of this disease.

The success of future chemoprevention strategies for lung cancer will require the following features: molecular targets whose modulation leads to a risk reduction, targeted agents that are effective either alone or in combination, and identification of high-risk populations (defined, at least in part, by the molecular targets). To date, most large, lung cancer prevention trials have not featured any of these attributes, and have instead employed pleiotropic natural agents in populations defined largely by smoking status. Some studies have evaluated the utility of integrating serial lung tissue assessments via bronchoscopic biopsies into lung-cancer-risk models, but have found no epithelial changes that increase the accuracy of risk prediction. As new, laboratory biomarkers are discovered and assessed in bronchial tissue, a combined approach that uses both histology and these biomarkers

\section{...future lung cancer chemo- prevention \\ trials are \\ likely to fail \\ unless they \\ incorporate a \\ highly defined \\ risk population \\ and reliable \\ biomarkers.}

ES Kim is Assistant

Professor in the

Department of

Thoracic/Head

and Neck Medical

Oncology, The

University of Texas

MD Anderson Cancer

Center, Houston, TX,

USA.

\section{Competing interests}

The author declared no

competing interests.

www.nature.com/clinicalpractice doi:10.1038/ncponc1293 will need to be completed with clinical data from patients.

Genetic susceptibility and environmental factors interact to influence carcinogenesis. Thus, combinations of multiple biomarkers and clinical characteristics might provide an effective riskassessment model; several lung cancer chemoprevention trials have attempted to incorporate this approach. ${ }^{8}$ In contrast to early, large-scale, prevention studies that used a nonspecific treatment and analyzed an end point of tumor recurrence or second primary tumor development, contemporary studies have now become smaller and focused on biomarkers; serial tissue and serum assessments are required to measure the biological effectiveness of the compound on biomarkers and epithelium.

Chemoprevention remains a very promising approach to help control lung cancer, yet future lung cancer chemoprevention trials are likely to fail unless they incorporate a highly defined risk population and reliable biomarkers. Celecoxib modulates the proliferation marker $\mathrm{Ki}-67$ in bronchial tissue. Serial biopsy assessments via bronchoscopy will create a rich resource of tissue in which to discover novel biomarkers. Small, biomarker-focused studies, such as these celecoxib trials, must be conducted before embarking on further, large-scale trials. 9,10 Once a clinical model of lung-cancer risk has been defined, such as that proposed by Spitz and coauthors, ${ }^{11}$ it can serve as a platform for the assessment of novel, targeted preventive agents. Their model was initially based on epidemiological and clinical data but has now been improved and refined by the addition of two markers of DNA repair capacity. Incorporation of lessons learned from our previous experience should allow us to improve lung cancer chemoprevention, which could lead to a reduction in the incidence and mortality of this devastating disease.

Supplementary information in the form of a reference list is available on the Nature Clinical Practice Oncology website. 